\title{
Ein mittelbronzezeitliches Schwert vom Typ Göggenhofen zwischen Bratislava und Wien aus Witzelsdorf in Niederösterreich
}

\section{A Middle Bronze Age sword type Göggenhofen between Bratislava and Vienna, from Witzelsdorf in Lower Austria}

\author{
Gerhard Trnka
}

\begin{abstract}
Abstrakt
Vor einem halben Jahrhundert wurde bei Witzelsdorf im Osten Österreichs ein Vollgriffschwert vom Typ Göggenhofen gefunden, das erst seit einigen Jahren der Forschung zugängig gemacht wurde. Es ist eine typische Schwertform der mittelbronzezeitlichen Stufe BZ C1, welche erstmals aus dem Gebiet der mitteldanubischen Hügelgräberkultur bekannt ist. Die Arbeit richtet sich auf eine eingehende Beschreibung, eine röntgenologisch und gußtechnische Untersuchung und eine zeitlich-kulturelle Einordnung.
\end{abstract}

\section{Schlagwörter}

Mittelbronzezeit, Hügelgräberkultur, Bronzezeit C1, Vollgriffschwert Typ Göggenhofen, Niederösterreich

\begin{abstract}
It is already fifty years ago that a full-grip sword of Göggenhofen type was found near Witzelsdorf in eastern Austria. But the find was not released for study until a few years ago. It is a typical sword form of the Middle Bronze Age phase BZ C1, which is first known from the territory of the Middle Danube Tumulus Culture. The paper is focused on a detailed description, $\mathrm{X}$-ray and metallurgical examination, as well as chronological and cultural classification.
\end{abstract}

\section{Keywords}

Middle Bronze Age, Tumulus Culture, Bronze Age C1, full-grip sword type Göggenhofen, Lower Austria 
Die mittlere Stufe der Hügelgräberkultur im Raum von der Ostschweiz über Südwest- und Süddeutschland bis Salzburg und Oberösterreich wird als „Stufe Göggenhofen“ bezeichnet (Müller-Karpe 1974, 14, Abb. 1). Namengebend ist das im Jahr 1911 in Göggenhofen (etwa 25 $\mathrm{km}$ südöstlich von München in Oberbayern) gefundene Hügelgrab eines Mannes, dessen erhaltene Bronzeausstattung aus einem Vollgriffschwert, einem Griffplattendolch, einer Petschaftkopfnadel, einem Armring mit Spiralenden, einem tordierten Armreif und einer Pinzette bestand (zusammenfassend von Quillfeldt 1995, 11, 35; Archäologie in Bayern 2006, Abb. 13). Dieses Fundensemble war auch eines jener Inventare, welche Paul Reinecke zur Definition seiner mittelbronzezeitlichen Stufe Bronzezeit C1 („Göggenhofen-Leibersberg“) in seinem bis heute für Mitteleuropa gültigen, alphabetisch geordneten Chronologiesystem der Bronzezeit (Reinecke 1924, 44) benutzte.

Zwei Inventare des Grabes haben auch eine nomenklatorische Bedeutung erlangt. Es ist dies zum einen die in der Mittelbronzezeit weit verbreitete und langlebige Petschaftkopfnadel vom Typ Göggenhofen (zusammenfassend Řihovský 1979, 59-62) und zum anderen das Vollgriffschwert vom Typ Göggenhofen, welches neben der kennzeichnenden Griffverzierung vor allem durch den im Querschnitt ovalen, ungekanteten Vollgriff (daher auch „Schwert mit ovalem, nicht gekantetem Vollgriff“" nach Friedrich Holste 1953, 19) gekennzeichnet ist.

Obwohl es nicht viele Exemplare dieser Schwerter (inklusive seiner Variante Trochtelfingen, bei dem die Griffstange im unteren Bereich einen schwach sechskantigen Querschnitt aufweist) gibt, ist deren Verbreitung in einem größeren Rahmen aus der Ostschweiz, Württemberg, Südbayern/Oberbayern und Mittelböhmen (Winiker 2015, 27-29) bekannt, ohne daß Werkstätten dieser Form auszumachen wären (von Quillfeldt 1995, 37-38, Tafel 111: A).
Somit war auch im ostösterreichischen Raum und den angrenzenden Gebieten diese Schwertausformung innerhalb der mitteldanubischen Hügelgräberkultur unbekannt (Krämer 1985, 12-13; Sicherl 2004, 37-38). Diesen ,fundleeren' Raum können wir nunmehr mit einem vor fast einem halben Jahrhundert gemachten Einzelfund eines Göggenhofener Schwertes bei Witzelsdorf (Abb. 1), $25 \mathrm{~km}$ östlich von Wien oder genau in der Mitte zwischen Bratislava und Wien (Abb. 2), auffüllen (Trnka 2016).

\section{Beschreibung: Vollgriffschwert aus Bronze (Abb. 3$)^{1}$}

Das Schwertfragment ist in einem sehr guten Erhaltungszustand und in seinem vorderen Klingenbereich zur Spitze hin alt gebrochen und dürfte ursprünglich eine Länge von 50-55 $\mathrm{cm}$ besessen haben. Die erhaltene Länge beträgt 37,4 cm, sein Gewicht 551,9 Gramm.

Die parallelseitige Klinge verjüngt sich leicht zur Spitze und weist abgesetzte Schneiden auf. Der Klingenquerschnitt ist flachoval. Das Röntgenbild zeigt, daß die Griffzunge gut im Griff eingesetzt ist (Abb. 4). Der Griff mißt 10,65 cm von den Heftflügeln bis zur Knaufspitze. Die Griffbefestigung erfolgte mittels zweier Nietstifte an der Klinge.

Das Heft mit schwach gewölbten Schultern schließt mit leicht geschwungenen Heftflügeln schräg zur Klingenmitte ab. Der Heftausschnitt ist dreiviertelkreisförmig und unverziert. Am Heft bzw. an den Heftflügeln sind über den zwei Befestigungsnieten vier Scheinnieten angebracht. Die gerade/parallelseitige Griffstange mißt samt Knaufplatte $6,55 \mathrm{~cm}$ und ist von spitzovalem Querschnitt. Ihre Breite beträgt 2,7 cm, ihre Dicke 1,65 cm. Auf der spitzovalen Knaufplatte $(5,3 \times 3,9 \mathrm{~cm})$ befindet sich der ovale, 1,8-1,5 × 0,7 cm messende Abschlußknopf. Im Gegensatz zur Klinge ist der Griff reich 


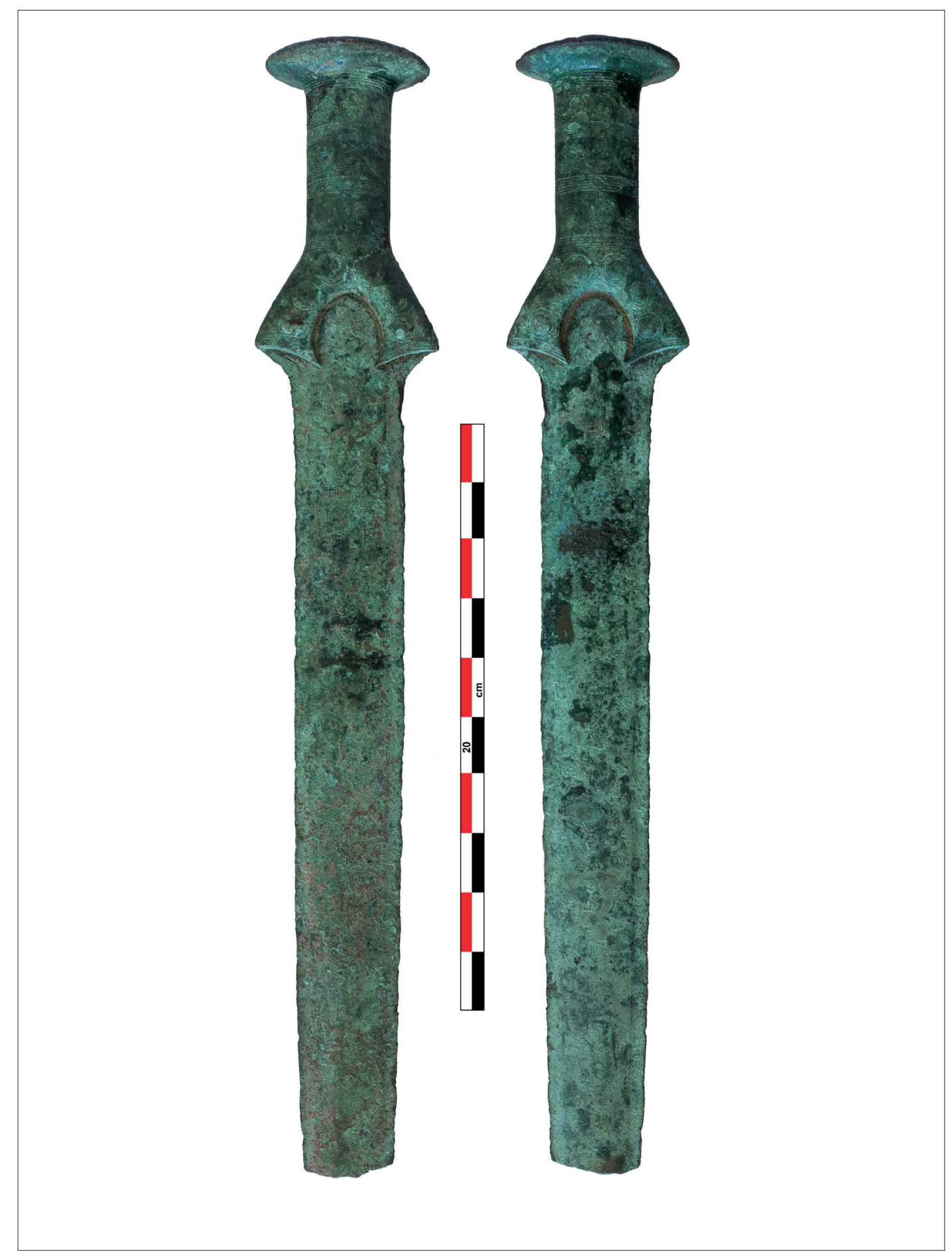

Abb. 1. Vollgriffschwert von Witzeldorf, Niederösterreich. Foto Gerhard Trnka. 


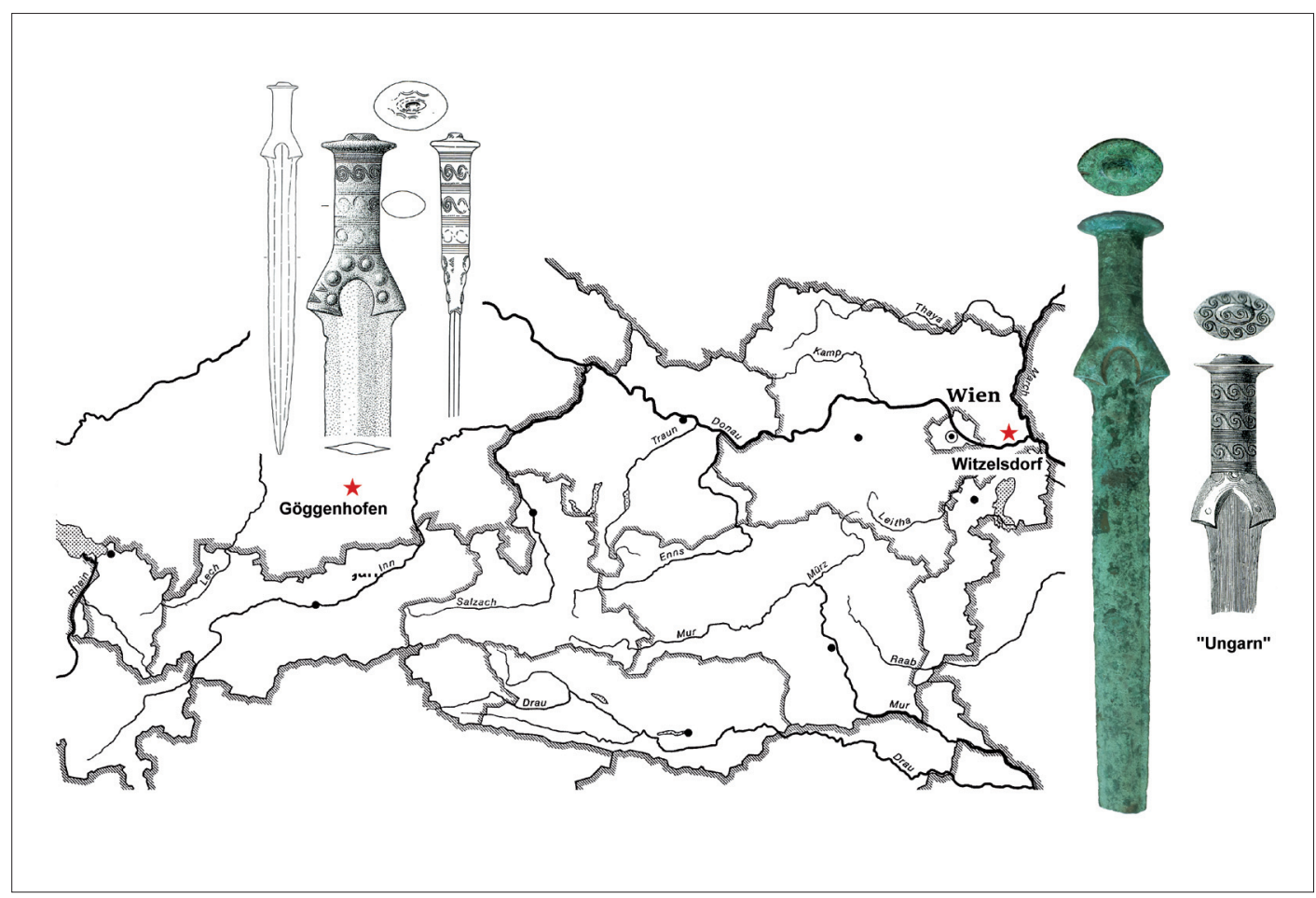

Abb. 2. Fundstellen Witzelsdorf (Niederösterreich), Göggenhofen (Oberbayern) und "Ungarn". Göggenhofener Schwert nach von Quillfeldt 1995, Tafel 2: 5; Schwert von „Ungarn" nach Hampel 1887, Tafel 21: 2.

verziert. Die Heftschultern werden auf beiden Seiten jeweils von einer Linie ausgehend von schraffierten Dreiecken eingefaßt, wobei in der Seitenansicht an diese Linien noch ovale Halbkreisbögen angebracht sind. Die Heftflügel schließen durch drei Linien ab. Die beiden Nieten an den unteren Heftflügeln sind durch zweifache Kreisaugen, die vier Scheinnieten durch dreifache Kreisaugen eingefaßt, wobei die beiden oberen Scheinnieten eventuell dicht gesetzte Punktreihen zeigen. Die Griffstange ist durch vier Linienbündel, welche aus sechs umlaufenden Linien bestehen, gegliedert. Die drei dazwischenliegenden Zonen sind beiderseits durch drei fortlaufende Spiralen gefüllt. Das obere Linienbündel weist zur Knaufplatte noch ein Band von ovalen Halbkreisbögen auf. Die Knaufplatte zeigt auf ihrer Oberseite einen mit einer Linie eingefaßten Rand, von dem zwölf Bogenfelder, die aus zwei und vier Linien bestehen, weggehen. Im Inneren der Bögen befinden sich jeweils zweifache Kreisaugen. Der ovale, gerade abschließende Knaufknopf weist über der Knaufplatte drei umlaufende Linien mit einem abschließenden, ovalen Halbkreismuster auf. Quer auf dem Knaufknopf verläuft ein Band aus vier Linien.

Die Oberfläche der Bronze ist teilweise hellgrün, an manchen Stellen auch dunkelgrün patiniert. Es sind nur geringe rezente Beschädigungen auf der Oberfläche und an den Schneiden festzustellen. 


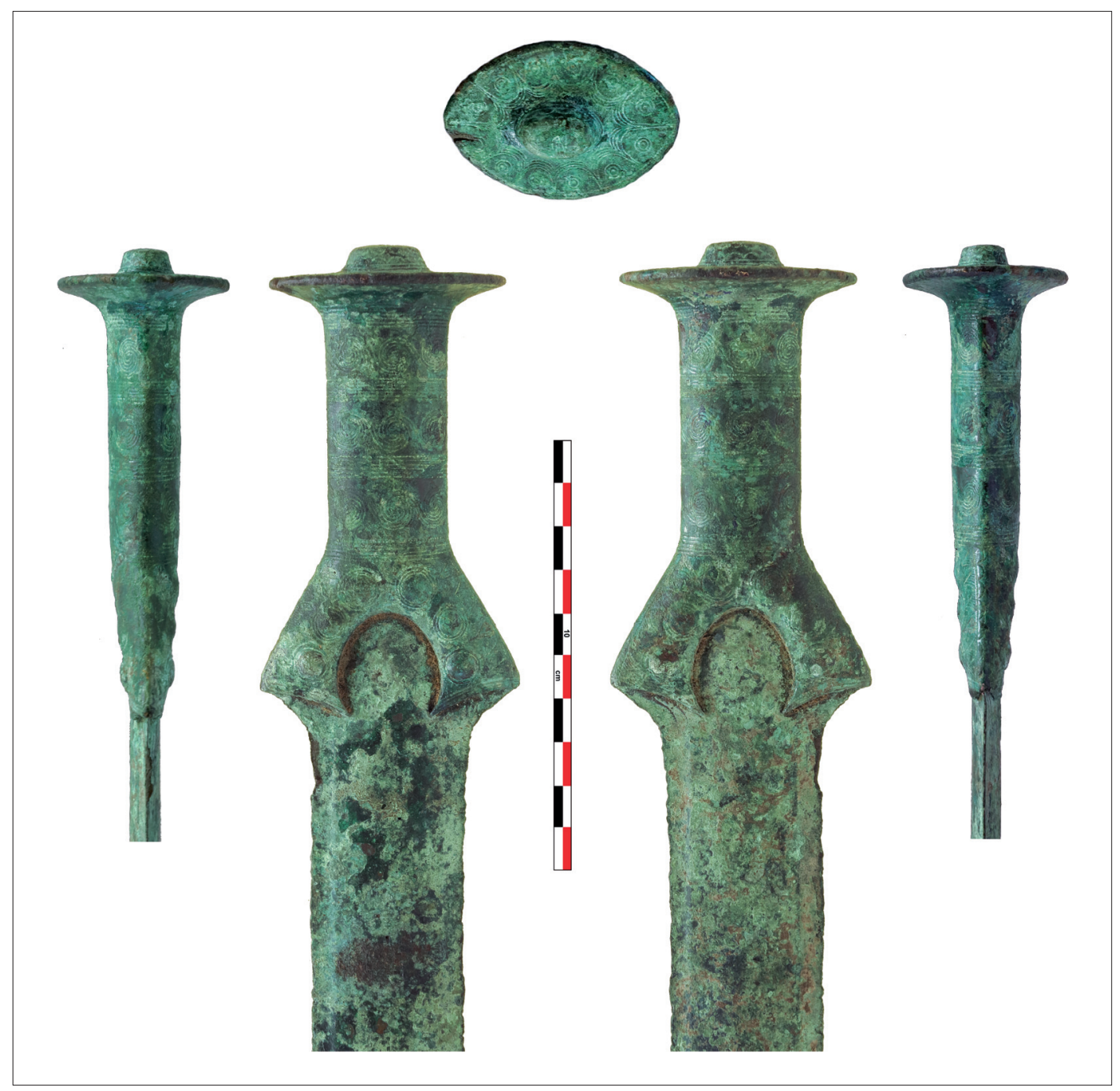

Abb. 3. Vollgriffschwert von Witzeldorf, Niederösterreich. Griff- und Klingenansatz. Foto Gerhard Trnka.

\section{Röntgenanalyse (Abb. 4)}

Das Röntgenbild zeigt, daß der Guß der Klinge aufgrund zahlreicher Lunker als schlecht zu bewerten ist. Da die Spitze fehlt, kann nicht entschieden werden, ob der Guß von dieser her erfolgte, was in der Regel bei (mittel)bronzezeitlichen Schwertern üblich ist (Mödlinger 2009, Abb. 1; Mödlinger 2011, 24-35, bes. 30-35, 98). In den separat gegossenen Griff wurde die Klinge (Heft und Griffzunge) eingepaßt und an den Flügelenden des Heftes mittels zweier Nietstifte befestigt. Bisherige Untersuchungen an bronzezeitlichen Vollgriffschwertern ergaben, daß es sich ausschließlich um Tüllengriffe handelte, deren (Lehm)Gusskern vollständig entfernt wurde. Die Vollgriffe wurden wahrscheinlich im Wachsausschmelzverfahren in einer Ton(guß)form erzeugt (Mödlinger 2011, 31). 
Trnka

Ein mittelbronzezeitliches Schwert vom Typ Göggenhofen zwischen Bratislava und Wien aus Witzelsdorf ...

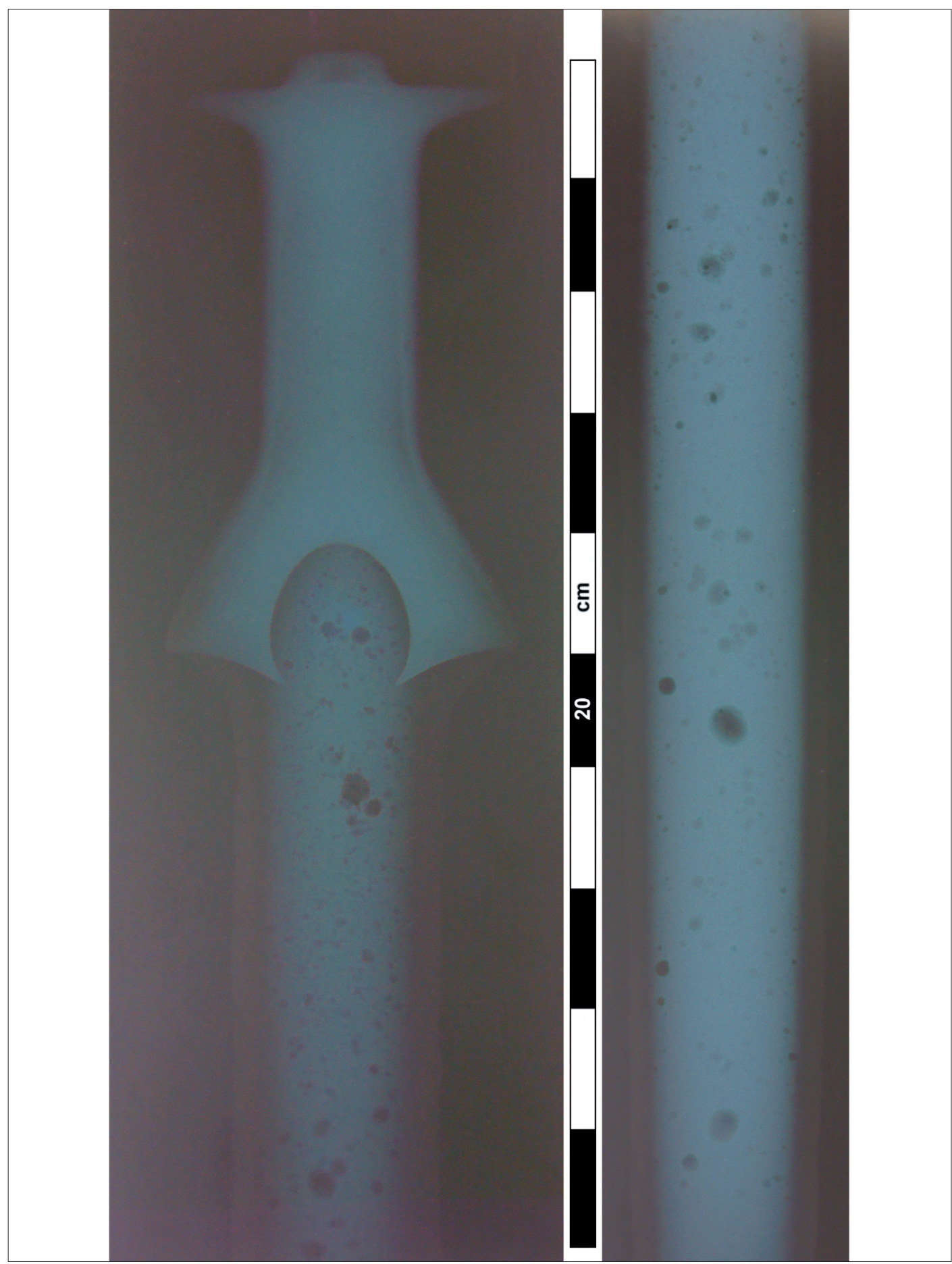

Abb. 4. Vollgriffschwert von Witzeldorf, Niederösterreich. Röntgen Institut für Urgeschichte und Historische Archäologie der Universität Wien. 
3. Kulturelle Stellung und Datierung

Klingenform und Griffgestalt weisen das Bronzeschwert von Witzelsdorf als mittelbronzezeitliche Stichwaffe aus. Die Griffmerkmale lassen eine Übereinstimmung mit den Vollgriffschwertern vom Typ Göggenhofen erkennen. Das namengebende Schwert aus dem Göggenhofener Grab in Oberbayern (siehe Archäologie in Bayern 2006, Abb. 13; Holste 1953, 19, Taf: 6: 6-11; Hahnekamp 2010) wird wie bereits umschrieben, in die mittlere Hügelgräberkultur bzw. die Stufe Bronzezeit C1 (Göggenhofener Stufe im süddeutschen/südbayerischen Raum) datiert und steht somit typologisch zwischen dem Typ Spatzenhausen (Bronzezeit B) und dem Achtkantschwert (Bronzezeit C).

Mit dem Witzelsdorfer Schwert scheint nunmehr auch Ostösterreich als Verbreitungsgebiet dieser Schwertausformung auf. Dazu mag auch als „östlichster Ausreißer“ ein nicht mehr vorhandenes Schwert aus dem ehemaligen - weit aufzufassenden Begriff - „Ungarn“ (Kemenczei 1991, 23, Tafel 11: 59) hinzugerechnet werden (siehe Abb. 2).

Zur Frage der Fundumstände des Witzeldorfer Schwertes als Einzelfund liegen keine Angaben vor. Obwohl die Fundstelle nahe zu den ehemaligen Altarmen der Donau liegt, zeigt die Patinierung keinerlei Anzeichen einer Lagerung in einem Feuchtbodenmilieu. Die Möglichkeit eines Grabfundes ist nicht auszuschließen, zumal in/ab der Göggenhofener Stufe das Aufkommen von Vollgriffschwertern in Männergräbern der „Oberschicht der zweiten Hälfte des 15. Jahrhunderts v. Chr.“ (i.e. Bronzezeit C1 bzw. Göggenhofener Stufe; Uenze 2012, 53) festzustellen ist, womit auch unser Schwert chronologisch innerhalb der mitteldanubischen Hügelgräberkultur einzuordnen ist. Der Zeitpunkt, an dem die Klinge abgebrochen wurde, läßt sich nicht mehr eruieren.

1) Aufgrund der Metallanalysen im Heftbereich der Klinge und des Griffes durch Mathias Mehofer (VIAS - Vienna Institute for Archaeological Science), besteht das Schwert aus einer Zinnbronze mit ca. 10-11\% Zinnkonzentration (Zinnanteil). Untersucht wurde das Schwert mittels eines Rasterelektronenmikroskopes der Firma Zeiss EVO 60 XVP mit angeschlossenem Analysesystem der Firma Oxford Instruments INCA 400. Für die Übermittlung der Analysenergebnisse möchte ich mich bei Mathias Mehofer herzlich bedanken. 


\section{Literaturverzeichnis}

Archäologie in Bayern 2006: Archäologie in Bayern Fenster zur Vergangenheit: Hrsg. von der Gesellschaft Archäologie in Bayern e. V., in Verbindung mit dem Bayerischen Landesamt für Denkmalpflege (Zusammengestellt von Sebastian C. Sommer), Regensburg.

Hahnekamp, Ch. 2010: http://chc.sbg.ac.at/schwerter/ map.php?filter\%5B0\%5D=g\%C3\%B6ggenhofen

Hampel, J. 1887: Alterthümer der Bronzezeit in Ungarn. Budapest.

Holste, F. 1953: Die bronzezeitlichen Vollgriffschwerter Bayerns. Münchner Beiträge zur Vor- und Frühgeschichte 4, München.

Kemenczei, T. 1991: Die Schwerter in Ungarn II (Vollgriffschwerter). Prähistorische Bronzefunde IV/9, Stuttgart.

Krämer, W. 1985: Die Vollgriffschwerter in Österreich und der Schweiz. Prähistorische Bronzefunde IV/10, München.

Mödlinger, M. 2009: Herstellung und Verwendung bronzezeitlicher Schwerter in Österreich. In: Krenn-Leeb, A. - Beier, H.-J. - Claßen, E. - Falkenstein, F. - Schwenzer, S. (Hrsg.): Mobilität, Migration und Kommunikation in Europa während des Neolithikums und der Bronzezeit. Varia Neolithica V - Beiträge zur Ur- und Frühgeschichte Mitteleuropas 53, Langenweissbach, 181-188.

Mödlinger, M. 2011: Herstellung und Verwendung bronzezeitlicher Schwerter aus Österreich. Eine vertiefende Studie zur mittelbronze- und urnenfelderzeitlichen Bewaffnung und Sozialstruktur.
Universitätsforschungen zur prähistorischen Archäologie 194, Bonn.

Müller-Karpe, H. 1974: Zur Definition und Benennung chronologischer Stufen der Kupferzeit, Bronzezeit und älteren Eisenzeit, Jahresber. Inst. Vorgesch. Univ. Frankfurt a.M. 1974, Frankfurt am Main, 7-18.

Reinecke, P. 1924: Zur chronologischen Gliederung der süddeutschen Bronzezeit, Germania 8, 43-44.

Řihouský, J. 1979: Die Nadeln in Mähren und dem Ostalpengebiet (von der mittleren Bronzezeit bis zur älteren Eisenzeit), Prähistorische Bronzefunde XIII/5, München.

Sicherl, B. 2004: Studien zur mittelbronzezeitlichen Bewaffnung in Tschechien, dem nördlichen Niederösterreich und der südwestlichen Slowakei. Universitätsforschungen zur prähistorischen Archäologie 107, Bonn.

Trnka, G. 2016: Ein mittelbronzezeitliches Vollgriffschwert aus Witzelsdorf, Niederösterreich, Archäologie Österreichs 27/1, 2016, 16-19.

Uenze, H. P. 2012: Bronzezeitliche Grabfunde von Unterach, Gemeinde Rehling, Landkreis AichachFriedberg (Schwaben), Bayerische Vorgeschichtsblätter $77,42-54$.

von Quillfeldt, I. 1995: Die Vollgriffschwerter in Süddeutschland, Prähistorische Bronzefunde IV/11. Stuttgart.

Winiker, J. 2015: Die bronzezeitlichen Vollgriffschwerter in Böhmen, mit einem Beitrag von Harry Wüstemann und Bernhard Sicherl, Prähistorische Bronzefunde IV/19. Stuttgart.

\section{ao. Univ.-Prof. Dr. Gerhard Trnka}

- Institut für Urgeschichte und Historische

Archäologie der Universität Wien

Franz-Klein-Gasse 1, A-1190 Wien

gerhard.trnka@univie.ac.at 